\title{
Depolarization ratio of low-frequency hydrogen bond Raman bands for semicrystalline pyrazinamide
}

\author{
A K KALKAR*1, N M BHOSEKAR ${ }^{1}$ and S T KSHIRSAGAR ${ }^{2}$ \\ ${ }^{1}$ Physics Division, Department of Chemical Technology, University of Bombay, Bombay \\ 400019 , India \\ ${ }^{2}$ National Chemical Laboratory, Pune 411003 , India

\begin{abstract}
The first measurements of the depolarization ratios for low frequency Raman active rotational and translational modes of semicrystalline (melt) pyrazinamide (PA) are reported. Tentative assignments have been proposed for the lattice modes based on polarization characteristics and their experimental intensities. Intra- and inter-translational oscillations have been assigned using an isolated single-chain approximation whereas the rotational oscillations are identified by their intensities and energy-order in similar NH...N hydrogen bond system. The PA dimer symmetry based on these results has been discussed and supports the planar conformation.
\end{abstract}

Keywords. Low-frequency Raman spectra; depolarization ratios; lattice vibrations; inter/ intramolecular hydrogen bond; pyrazinamide dimer.

\section{Introduction}

Solid pyrazinamide (PA) forms a dimer through intermolecular (NH...H) hydrogen bonding. The $\mathrm{X}$-ray analysis of this dimer at room temperature is well-documented and indicates an almost close planar pyrazine ring and carboxamide group, which itself is planar with a slight deviation of the order of $3.2^{\circ}$ from the plane of the ring. However, anomalies are observed in IR (Kalkar et al 1989) and X-ray (Takaki et al 1960) analyses regarding the most favourable potential site in PA for forming the dimer. Infrared studies favour the dimer geometry illustrated in figure 1.

The purpose of the present study is to discuss the possible structure of the PA dimer in the light of the comparative study of the polarization behaviour of these relevant low frequency lattice modes originating out of the dimer structure in the pure glassy cooled semicrystalline (melt) solid. (Here by semicrystalline we mean that it is not a single crystal.) Nevertheless, in the absence of single crystal data such a discussion could at least lead us to tentative conclusions regarding the PA dimer structure reflected through vibrational lattice modes. However, the spectral analysis has been proposed on the basis of single crystal symmetry considerations.

\footnotetext{
* For correspondence
} 


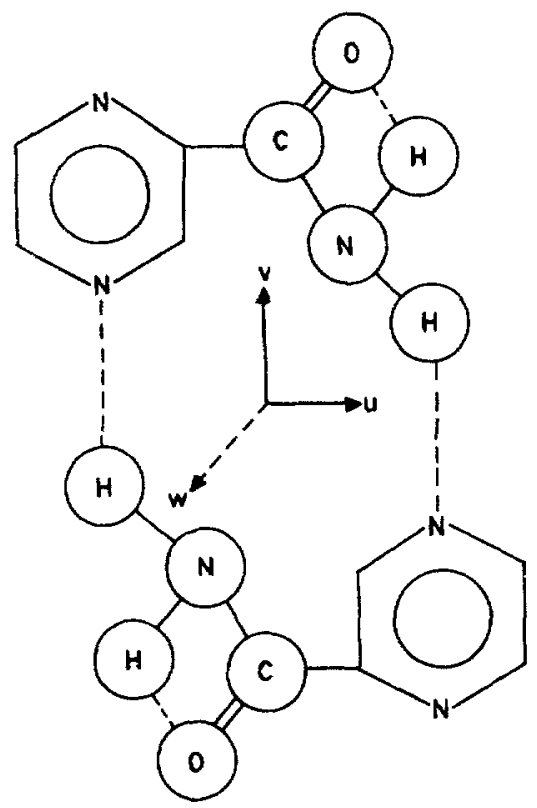

Figure 1. Pyrazinamide-dimer geometry.

\section{Experimental}

All Raman measurements $\left(10-200 \mathrm{~cm}^{-1}\right)$ were recorded with a Spex-1403 double monochromator spectrometer attached to a Spex-1442 third monochromator for high performance of the spectrometer. The scattered radiation was collimated by a Minolta lens ( $55 \mathrm{~mm}, f / 1.2)$ in photon counting mode using a C-31034 GaAs photomultiplier-detector system. The Spex DMI-Datamate spectrometer controller and data processor were used for data processing.

The 514.5 mm excitation radiation from an $\mathrm{Ar}^{+}$laser Spectra Physics 165-08, using a Spex-lasermate filter, was focused on the sample. A cylindrical lens was used to focus the laser beam. The intensity of the radiation incident on the sample was of the order of $250 \mathrm{~mW}$. The incident beam was polarized in either the vertical or the horizontal plane while the analyser near the spectrometer slit was kept fixed for horizontal polarization during all measurements. The polarization ratio values determined under such conditions, $\rho_{P}=I_{1} / I_{\|}$, are in the range $\left(0 \leqslant \rho_{P}<3 / 4\right)$ for totally symmetric and $\left(\rho_{p} \geqslant 3 / 4\right)$ for non-totally symmetric vibrations (Nakamoto 1986). The reproducibility of depolarization $\left(\rho_{P}\right)$ data, in the present experiments, is of the order of \pm 0.02 .

The capillary-packed cooled melt (semicrystalline) PA sample, a clear glass without any distortion, obtained from M/s Aldrich U.S.A., was used for low frequency Raman spectra recordings. Keeping instrumental conditions the same, several scans were recorded and stored. The final traces were obtained as a average of stored data (figure 2). The spectrum quality is satisfactory and well-resolved, which facilitate depolarization measurements. The overall reproducibility of the spectrum and the intensities of absorptions, in particular, are excellent and within $\pm 2 \%$. 

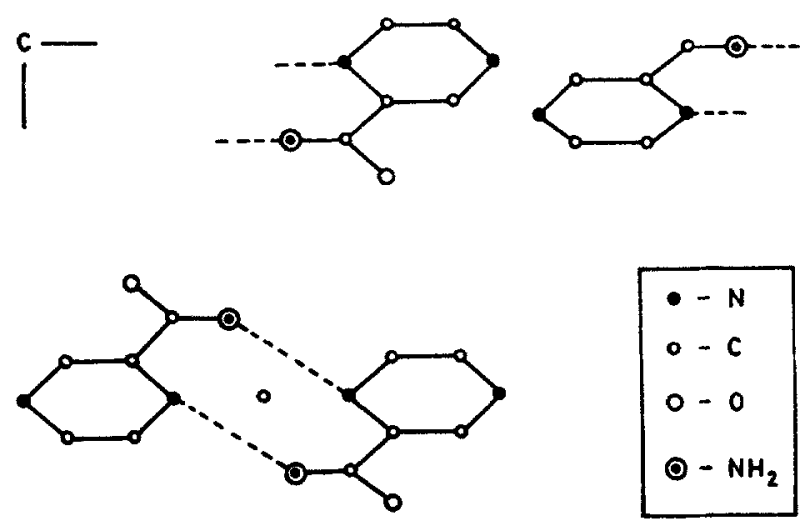

Figure 2. Four-molecule arrangement in crystal lattice.

\section{Results and discussion}

\subsection{Classification of vibrations}

PA molecules crystallize in the monoclinic system space group, $P 2_{1} / a-C_{2 h}^{5}$, with four molecules in the unit cell (figure 3) (Takaki et al 1960), giving rise to twenty-one lattice vibrations. Nine $(3 A g+2 A u+1 B u+3 B g)$, out of totally twenty-one lattice vibrations $(6 A g+5 A u+4 B u+6 B g)$, are translational modes and essentially belong to intra (dimer) and inter (dimer-dimer)-chain $\mathrm{NH}$...H hydrogen bond vibrations. The remaining twelve vibrations $(3 A g+3 A u+3 B u+3 B g)$ are rotational oscillations.

3.1a Translational vibrations: In order to distinguish between the two types of translation modes, the isolated single chain approximation has been used (Millikan and Pitzer 1958; Perchard and Novak 1968; Colombo et al 1971), as the forces between neighbouring molecules in a chain are much stronger than the inter-chain forces. Table 1 represents the correlation diagram between the chain repeat unit $(C s)$ and crystal lattice $\left(C_{2 h}\right)$.

The corresponding factor group for the dimer PA chain is $C s$ with the $\sigma c$ glide plane parallel to the $C$-axis as the only symmetry plane. With two molecules per repeat unit there are eight intra-chain vibrations, involving the hydrogen bond equally divided in symmetrical $A^{\prime}\left(1 T^{\prime}\right.$ and $\left.3 R\right)$ and antisymmetrical $A^{\prime \prime}\left(2 T^{\prime}\right.$ and $\left.2 R^{\prime}\right)$ with respect to the symmetry plane. Out of eight intra-chain hydrogen bond stretching vibrations, one is derived from the translation out-of-plane motions with respect to the symmetry glide plane and other from rotational nature. Both belong to the $\boldsymbol{A}^{\prime \prime}$ species. The bending vibrations are due to other translations $\left(1 A^{\prime}\right.$ and $\left.1 A^{\prime \prime}\right)$ and rotational $\left(1 A^{\prime \prime}+2 A^{\prime}\right)$ motions.

A lone, $A^{\prime}$ species rotational motion, is predominantly torsional motion about the chain axis in nature. In the unit cell $\left(C_{2 h}\right)$ factor group $A^{\prime}$ and $A^{\prime \prime}$ vibrations are derived from $B u$ and $A u$ crystal species respectively except a chain-axis rotation $A u$ vibration which is overlooked in the single-chain $(C s)$ considerations. However, of the six hydrogen bond vibrations which appear in the $C_{2 h}$ factor group, three each originating from $A g\left(A^{\prime}\right)$ and $B g\left(A^{\prime \prime}\right)$ species are Raman-active due to the space group inversion centre. 

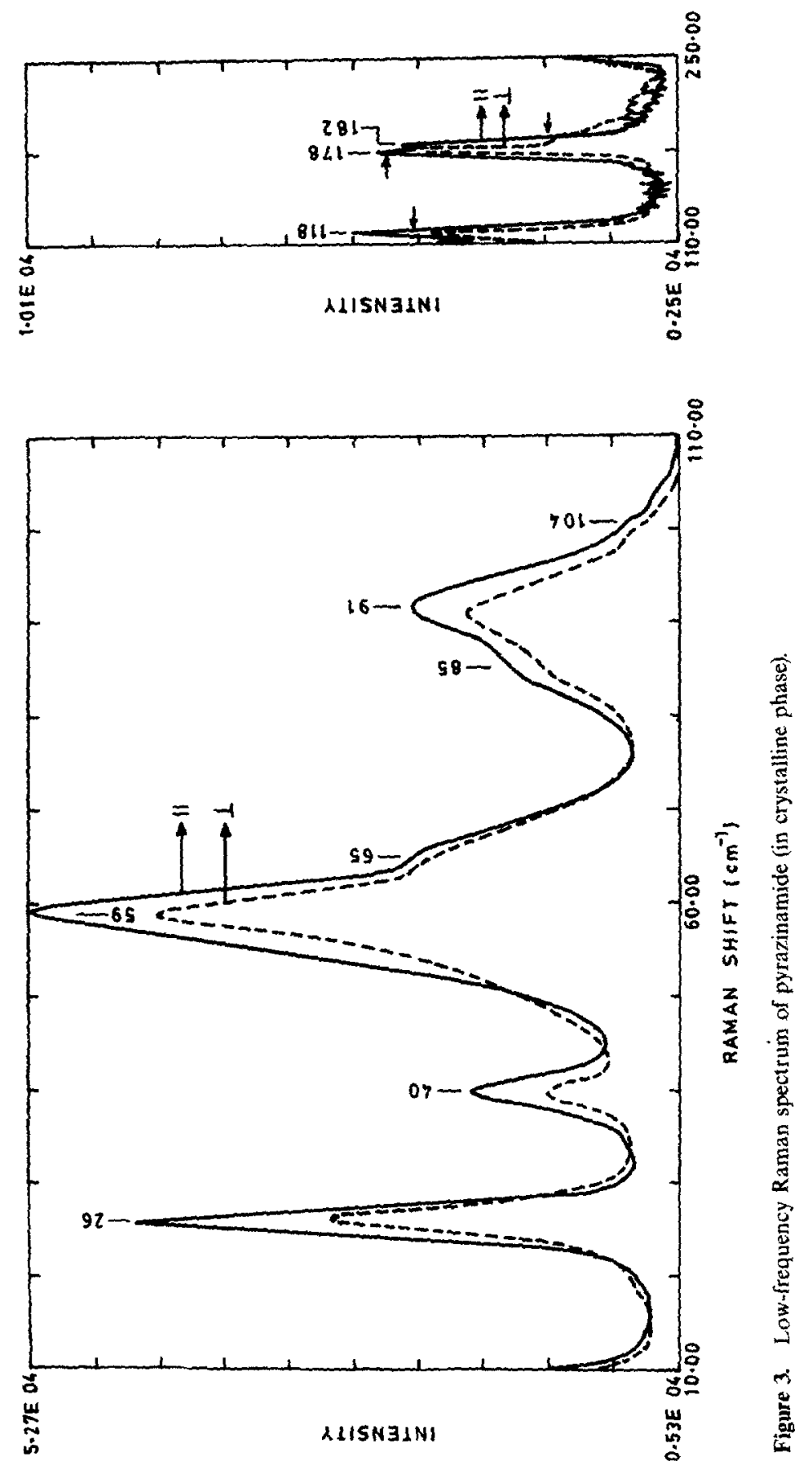
Table 1. Correlation diagram between the point group of monomers, dimers and the lattice space group of pyrazinamide.

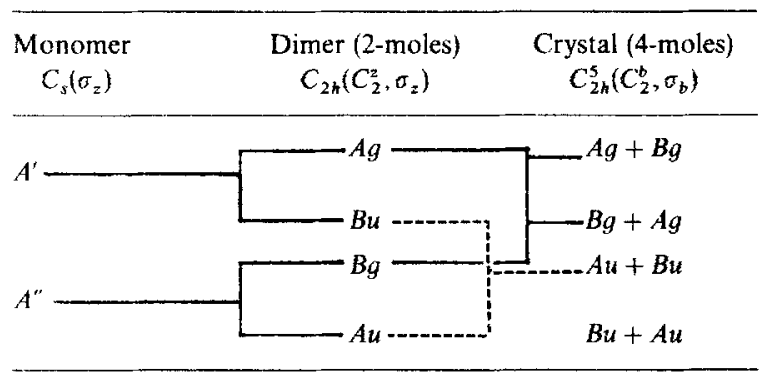

3.1b Rotational vibrations: The definite identification of rotational vibrations is difficult in the present case. However, the present assignments are based on the assumption that the perturbed rotational oscillations due to hydrogen, are essentially high intensity absorption Raman bands with longer splitting in symmetrical and antisymmetrical modes (Colombo 1968). Out of twelve lattice rotational modes, six oscillations of symmetry $(3 \mathrm{Ag}+3 \mathrm{Bg})$ are Raman-active.

3.1c Assignments: The assignments of the observed bands to corresponding symmetry species are made using the polarization behaviour of these bands (table 2). The $A^{\prime}(A g)$ modes are expected to yield polarized Raman lines whereas the $A^{\prime \prime}(B g)$ mode should give rise to depolarized Raman lines. The labelling of intra- and inter-translational and rotational vibrations are based upon the symmetry and experimental intensity considerations. In Raman spectra inter-chain translation vibrations are low-energy low intensity bands rather than intra-chain motions due to the difference between the force constants involved (Colombo et al 1971). However, Raman-active rotational vibrations are generally stronger than all other lattice modes (Colombo 1968).

In low frequency Raman measurements, we observed eight predominant absorption bands at $184,178,118,104,91,59,40$ and $26 \mathrm{~cm}^{-1}$ with clear shoulders at 85 and $65 \mathrm{~cm}^{-1}$. The low intensity Raman bands at 184 and $178 \mathrm{~cm}^{-1}$ have the highest frequencies. The band at $184 \mathrm{~cm}^{-1}$ exhibits polarized character whereas that at $178 \mathrm{~cm}^{-1}$ is depolarized. Thus these bands are assigned to symmetry type $A q\left(A^{\prime}\right)$ and $B g\left(A^{\prime \prime}\right)$, respectively. The latter has been assigned to the Raman-active hydrogen-bond stretching of the $\mathrm{NH} \ldots \mathrm{H}$ intra-chain oscillator along the $\mathrm{C}$-axis of the lattice. The band at $184 \mathrm{~cm}^{-1}$, which is more intense than that at $178^{-1}$, has been proposed as due to $V$-axis symmetrical rotational motions.

Similarly, the high-energy band pair at 118 and $104 \mathrm{~cm}^{-1}$ are both of the symmetry type $B g\left(A^{\prime \prime}\right)$. The band at $104 \mathrm{~cm}^{-1}$ exhibits comparatively higher intensity than that at $118^{-1}$. On the basis of the higher intensity the band at $104 \mathrm{~cm}^{-1}$ has been assigned to $V$-axis asymmetrical rotation whereas $118 \mathrm{~cm}^{-1}$ band is proposed for intrachain bending motion.

In the mid-energy region, two bands at 91 and $85 \mathrm{~cm}^{-1}$ were observed. The band at $91 \mathrm{~cm}^{-1}$ is one of the strongest bands in the spectrum and is polarized in character. The high intensity of this band indicates that it is one of the Raman-active rotational motions (Colombo et al 1971). Thus, the band is attributed to the $U$ axis symmetrical rotational mode of $A g\left(A^{\prime}\right)$ symmetry. The other band at $85 \mathrm{~cm}^{-1}$ appears as a shoulder to the high intensity $91 \mathrm{~cm}^{-1}$ band. This shoulder at $85 \mathrm{~cm}^{-1}$ is predominant and 
Table 2. Hydrogen bond vibrations in the Raman spectrum of crystalline (melt) pyrazinamide.

\begin{tabular}{|c|c|c|c|c|}
\hline $\begin{array}{l}\text { Frequency } \\
\qquad\left(\mathrm{cm}^{-1}\right)\end{array}$ & Intensity & Symmetry & Polarization $\left(\rho_{P}\right)$ & Assignments \\
\hline \multicolumn{5}{|c|}{ Translational vibrations } \\
\hline - & -- & $B g, A^{\prime \prime}$ & - & $\left.T_{r \cdot}\left(\|_{b}\right)\right]$ \\
\hline 26 & $3 \cdot 3, s$ & $A g, A^{\prime}$ & $P, 0.62$ & $T_{r},\left(\|_{c}\right)$ \\
\hline-- & $\longrightarrow$ & $A g, A^{\prime}$ & - & translation \\
\hline 65 & $1 \cdot 4, m$ & $A g, A^{\prime}$ & $P, 0 \cdot 50$ & $T_{r}$, O.P. bending $\rceil$ Intra \\
\hline 104 & $0 \cdot 1, w$ & $B g, A^{\prime \prime}$ & $d p, 0-80$ & \begin{tabular}{l|l}
$T_{r}$, I.P. bending & chain
\end{tabular} \\
\hline 178 & $0 \cdot 31, w$ & $B g, A^{\prime \prime}$ & $d p, 0-82$ & $T_{r}$ stretching $\rfloor_{\text {translation }}$ \\
\hline \multicolumn{5}{|c|}{ Rotational vibrations } \\
\hline 40 & $0.95, m$ & $\operatorname{Ag}, A^{\prime}$ & $P, 0 \cdot 42$ & $R_{s}(W)$ \\
\hline 59 & $4 \cdot 3, s$ & $B g, A^{\prime \prime}$ & $d p, 0.78$ & $R_{a}(W)$ \\
\hline 85 & $1 \cdot 0, m$ & $A g, A^{\prime}$ & $P, 0 \cdot 56$ & $R_{s}(U)$ \\
\hline 91 & $1 \cdot 9, m$ & $B g, A^{\prime \prime}$ & $d p, 0.79$ & $R_{a}(V)$ \\
\hline 118 & $0.40, w$ & $B g, A^{\prime \prime}$ & $d p, 0.80$ & $R_{s}(V)$ \\
\hline 184 & $0 \cdot 31, w$ & $A g, A^{\prime}$ & $P, 0 \cdot 43$ & $R_{a}(V)$ \\
\hline
\end{tabular}

$P=$ polarised; $d p=$ depolarised; $\rho_{P}=$ depolarisation ratio; $s=$ strong; $m=$ medium; $w=$ weak; $R_{s}=$ symmetric rotation; $R_{a} \pm$ asymmetric rotation

clearly shows polarized character, and belongs to $\operatorname{Ag}\left(A^{\prime}\right)$ symmetry class. This band is attributed to the asymmetrical component of the $U$-axis rotational mode.

The Raman bands at $65,59,40$ and $26 \mathrm{~cm}^{-1}$ are low energy bands in the spectrum. However, the bands at 59 and $26 \mathrm{~cm}^{-1}$ are strongest in intensity whereas the $65 \mathrm{~cm}^{-1}$ band appears as a shoulder to the $59 \mathrm{~cm}^{-1}$ band. Except the band at $59 \mathrm{~cm}^{-1}\left(B g, A^{\prime \prime}\right)$, the other three are polarized and thus belong to the $A g\left(A^{\prime}\right)$ symmetry class. The lowest frequency $26 \mathrm{~cm}^{-1}$ is assigned to inter-chain translation motions of the lattice. The high intensity of the band indicates that it is arises due to the most favourable $C$-axis plane inter-chain lattice motions. The bands at 59 and $40 \mathrm{~cm}^{-1}$ are proposed for antisymmetric and symmetric $W$-axis rotational oscillations. The high intensity shoulder at $65 \mathrm{~cm}^{-1}$ exhibits $B g\left(A^{\prime \prime}\right)$ symmetry and is expected to belong to translational motion, either inter- or intra-chain. However, the intensity and energy factors favour the intra-chain out-of-plane bending motion. The proximity of this mode to the rotational one indicates the strong possibility of interaction, thus enhancing the $59 \mathrm{~cm}^{-1}$ band intensity.

\section{PA-dimer geometry}

The low frequency lattice vibrational analysis reveals that the PA molecule symmetry $\left(C_{2 h}\right)$ in the crystal is higher than in the free molecule $(C s)$, essentially due to the hydrogen bond chain formations. In the present case, as well as for free molecules (Kalkar et al 1989) the polarization behaviour, and accordingly the distribution of the respective models, supports the symmetry conclusions which are highly dependent on the frame geometry (Furic 1983). The NH...N hydrogen bond calculated by using - NH internal mode vibrations is $3.22 \AA$ which is comparable to $3.79 \AA$, the corresponding Van der Waals radii. The present results thus support the planar structure of the PA free dimer as well as endless chains in the crystal. 


\section{References}

Colombo L 1968 J. Chem. Phys. 494688

Colombo L, Furic K and Kirin D 1971 J. Mol. Spectrosc. 39217

Furic K 1983 Croatica Chem. Acta $\mathbf{5 6} 1$

Kalkar A K, Bhosekar N M and Kshirsagar S T 1989 Spectrochim. Acta A45 635

Millikan R C and Pitzer K S 1958 J. Am. Chem. Soc. 803515

Nakamoto K 1986 Infrared and Raman spectra of inorganic and coordination compounds 4th edn (New York: John Wiley and Sons) p. 74

Perchard C and Novak A 1968 J. Chem. Phys. 483079

Takaki Y, Sasada Y and Watanabe T 1960 Acta Crystallogr. 13693 